Itinéraires Itinéraires

Littérature, textes, cultures

\title{
Nouvelles expressivités littéraires pour L'Afrique qui vient : Alain Mabanckou et Léonora Miano
}

New Literary Expressivities for "the Africa to come" (L'Afrique qui vient): Alain Mabanckou and Léonora Miano

Josefina Bueno Alonso

\section{OpenEdition}

\section{Journals}

Édition électronique

URL : http://journals.openedition.org/itineraires/6030

DOI : 10.4000/itineraires.6030

ISSN : 2427-920X

Éditeur

Pléiade

Référence électronique

Josefina Bueno Alonso, « Nouvelles expressivités littéraires pour L'Afrique qui vient : Alain Mabanckou et Léonora Miano », Itinéraires [En ligne], 2019-1 | 2019, mis en ligne le 16 juillet 2019, consulté le 03 août 2019. URL : http://journals.openedition.org/itineraires/6030 ; DOI : 10.4000/itineraires.6030

Ce document a été généré automatiquement le 3 août 2019

\section{(1) $\odot \Theta \Theta$}

Itinéraires est mis à disposition selon les termes de la licence Creative Commons Attribution - Pas d'Utilisation Commerciale - Pas de Modification 4.0 International. 


\title{
Nouvelles expressivités littéraires pour L'Afrique qui vient : Alain Mabanckou et Léonora Miano
}

\author{
New Literary Expressivities for "the Africa to come" (L'Afrique qui vient): \\ Alain Mabanckou and Léonora Miano
}

Josefina Bueno Alonso

« L'on aura beau ériger des frontières, construire des murs, des digues et des enclos, diviser, sélectionner, classifier et hiérarchiser, chercher à retrancher de l'humanité ceux et celles que l'on méprise, qui ne nous ressemblent pas ou avec

lesquels nous pensons que nous n'avons, à première vue, rien en commun - il n'y a qu'un seul monde et nous en sommes tous les ayants droit. En

principe, ce monde nous appartient à tous également, et nous en sommes tous les héritiers même si les manières de l'habiter ne sont pas les mêmes - d'où justement la réelle pluralité des formes culturelles, des langages et des façons de vivre ». (Achille Mbembe, « Pièces d'identité et désirs d'apartheid », 2010, p. 115)

1 Cette citation du philosophe camerounais, promoteur de la pensée circulaire du monde ${ }^{1}$, montre l'irréversible emmêlement et entrelacement des cultures, des êtres et des choses. L'irruption de l'Autre, de l'Ailleurs, dans l'espace français, le bousculant, le transformant, l'enrichissant, n'est pas le résultat de ces dernières décennies, mais un fait depuis plus d'un siècle. C'est en 2010 que paraît son essai, Sortir de la grande nuit, dans lequel il évoque une vision particulière du cosmopolitisme qu'il définit comme une sensibilité culturelle, historique et esthétique nommée Afropolitisme $e^{2}$, désignant avant tout une façon d'être dans le monde contemporain. 
2 Notre analyse aborde les ouvrages critiques de deux écrivains qui participeraient à la mise en œuvre d'une Renaissance littéraire africaine à partir de leur militantisme dans leurs créations artistiques, de leurs espaces de diffusion et des liens qu'ils tissent avec la critique africaniste contemporaine. Même si les idées ne sont pas toujours inédites, le contexte d'énonciation, le statut des locuteurs et la nature des textes leur confèrent un potentiel de diffusion non négligeable (Imorou 2017 : 149). La façon de reprendre les idées est novatrice et le statut des écrivains est différent ${ }^{3}$. Le discours artistique des écrivains est le résultat d'un cheminement intellectuel, la pratique littéraire étant devenue une « philosophie de vie » (Miano 2016a : 67). J'ajouterai à l'ampleur médiatique, le fait que les écrivains et les penseurs africains s'insèrent dans les luttes sociales contemporaines (immigration, racisme, identité nationale, révision de la politique coloniale en France, etc.) renvoyant à une réflexion circulaire sur l'Autre et sur soi-même. En d'autres termes, l'écrivain noir s'érige au centre du débat politique et social sur les identités africaines. Il les interpelle et il en propose une nouvelle cartographie ainsi qu'une transformation des représentations et des institutions, entre autres, de l'institution littéraire.

Dans cette contribution, nous aborderons cette Afrique qui vient, dont la création artistique se veut être à la source. Quelle serait la contribution des essais de Mabanckou, tout particulièrement Penser et écrire l'Afrique aujourd'hui? Quel serait l'apport des essais de Miano à cette nouvelle $\mathrm{f}(\mathrm{F})$ rancophonie ${ }^{4}$ et à la réhabilitation de l'afrodescendance ? Faudrait-il y voir également un apport de genre au sein de la critique africaniste contemporaine ? Les réflexions de Mabanckou et de Miano rejoignent les lignes de pensée d'Achille Mbembe et de Felwine Sarr, l'écrivain dépasse les pourtours de la littérature pour prendre le rôle de divulgateur d'idées 5 .

\section{L'Afrique qui vient}

4 La critique africaniste prend un tournant à partir des réflexions théoriques publiées par des écrivains et intellectuels originaires d'Afrique subsaharienne. Leur réflexion sur les relations qu'ils entretiennent avec la langue française et avec une Francophonie institutionnelle est particulièrement intéressante. Des écrivains de la quatrième génération, «les enfants de la postcolonie ou la génération transcontinentale » (Waberi 2017 : 156) passent de la fiction à la réflexion théorique et se placent aux côtés de critiques et penseurs tels que Achille Mbembe ou Felwine Sarr élaborant un nouveau discours africain ${ }^{6}$ pour un continent à construire, qui doit s'écrire et qui doit être repensé. C'est le cas d'Alain Mabanckou et de Léonora Miano qui annoncent, sinon une rupture, en tout cas un changement par rapport à la génération précédente. Les deux auteurs partagent des traits communs : ils sont nés après l'année 1960, ils se considèrent comme africains mais veulent dépasser cette appartenance, ils sont récipiendaires de prix littéraires ${ }^{7}$ et le thème du retour au pays natal a disparu et c'est plutôt le thème contraire, le thème de l'Africain en France, qui traverse leurs fictions.

5 L'advenue de cette Afrique qui vient est un défi qui ressort avant tout de la création artistique, tout particulièrement la littérature (Mbembe 2017 : 28). Dans ce sens, de nombreuses publications à partir des années 2000 portent dans leur titre ou font allusion à cette Afrique qui vient. En 2013, Alain Mabanckou et Michel Le Bris, publiaient une anthologie de textes d'écrivains africains ${ }^{8}$, intitulée L'Afrique qui vient. Le continent a besoin de se redéfinir, il ne s'adapte plus aux discours établis ni aux stéréotypes qu'il a longtemps soufferts. L'Afrique qui vient est inquiétante et surprenante, écrit Alain 
Mabanckou. Nous sommes face à un continent qui échappe à la délimitation territoriale car étant pluriel et hétérogène, il est marqué par l'afropolitanisme qui naît de l'échange, des expériences, des rencontres, de la redéfinition et de la décentralisation de l'espace. Tels sont les aspects soulignés par Alain Mabanckou, dans Penser et écrire l'Afrique aujourd'hui (2017) ${ }^{9}$, volume qui rassemble les contributions d'africanistes qui se sont rencontrés en 2016 au Collège de France :

Penser l'Afrique, c'est se demander « quelles sont les grandes lignes de fracture ou encore les grands antagonismes qui nous donnent l'impression de vivre un moment particulièrement agité de l'histoire de notre monde ; qui nous donnent le sentiment inquiet d'être face à des choix irréconciliables, ou encore vivre une histoire qui se décline désormais sur le mode du désordre et du fracas "; c'est aussi [...] relever le défi de notre temps qui consiste à savoir « quelle langue et quelle écriture seront capables de restituer à l'Afrique sa force, sa puissance propre en même temps que sa figure-monde ». (Mabanckou $2017: 10,11$ )

6 Le discours contemporain sur l'Afrique qui vient se trouve divisé entre la foi d'un futur alléchant ${ }^{10}$, et d'un autre côté, un présent qui apparaît sous une apparence chaotique. À ce sujet, Felwine Sarr reprend deux concepts Afrotopos et Afrotopia, évocation d'une sorte d'utopie africaine, pour dessiner l'avenir du continent (Sarr $2016: 14$ ).

7 Les principaux enjeux du continent africain, un des continents les plus peuplés dans les prochaines décennies, sont les mouvements migratoires et la mondialisation. Tel que le remarque Achille Mbembe, bien que la mondialisation gouverne la planète, «la vieille catégorie politique de la souveraineté se confond désormais avec le droit de décider de qui peut bouger et à quelles conditions» (Mbembe 2017: 19). Une des principales conséquences de la mondialisation est que notre monde s'est considérablement réduit et que nous assistons à une crise de l'idée de frontière. Ce découpage en groupes socioculturels préconstruits et distincts les uns des autres donne naissance, dans les États-nations, à ce qu'Étienne Balibar et Immanuel Wallerstein appellent «l'ethnicité fictive » dans le sens où il s'agit d'une identité de «fabrication »(Balibar 1997 : 130-131).

En pleine ère de la mondialisation et de la révolution technologique, notre monde devient paradoxalement limité. Cette même critique se retrouve dans Le Sanglot de l'homme noir ${ }^{11}$, où Mabanckou tente de déconstruire les limites territoriales qui contribuent à l'association imaginaire d'une « identité nationale fixe et standardisée » :

Pour certains Français, il est difficile d'admettre que nombre de leurs compatriotes ne leur ressemblent pas et que l'idée d'une France blanche n'est plus de nos jours qu'une illusion, une image figée qui condamne celui qui aura acquis la nationalité française à passer sa vie à se justifier, à expliquer au citoyen "de souche "ses origines... [...] Ma conception de l'identité dépasse de très loin les notions de territoire et de sang. [...] L'histoire de la colonisation nous a montré que le territoire pouvait être imaginaire, dépasser les frontières, braver les variations climatiques, brasser les langues et les races. (Mabanckou 2012 : 58-59)

9 Pour la construction d'un nouveau concept de l'Afrique, Mbembe (2017) et Sarr (2016) revendiquent non seulement la pensée circulaire mais la recherche de paramètres de développement différents des modèles occidentaux; ils proposent de nouvelles clés d'interprétation de l'histoire-monde. La circulation des mondes, ainsi que la penséemonde, se retrouvent à l'origine de la nouvelle conception du continent africain. Sarr parle d'un des grands enjeux de l'Afrique, l'afrocontemporanéité (2016:41) qui permettra au continent de se réaffirmer dans ses différences fécondes, sans tomber dans le communautarisme ${ }^{12}$. Cette pensée circulaire implique une «transnationalisation de la société, de la vie intellectuelle et artistique », et c'est là le champ de travail des écrivains 
et intellectuels africains tels Mbembe, Mabanckou, Sarr ou Miano, en contribuant à la diffusion de la création d'un espace public afropolitain.

«L'Afrique, existe-t-elle?», «De quelle Afrique parlons-nous?», Alain Mabanckou répond de façon affirmative, loin d'un essentialisme nostalgique qui puisse contribuer à accentuer les préjugés sur les lettres africaines :

L'Afrique n'est plus seulement en Afrique. En se dispersant à travers le monde, les Africains créent d'autres Afriques, tentent d'autres aventures peut-être salutaires pour la valorisation des cultures du continent noir. Revendiquer une " africanité " est une attitude fondamentaliste et intolérante. L'oiseau qui ne s'est jamais envolé de l'arbre sur lequel il est né comprendra-t-il le chant de son compère migrateur? Nous avons besoin d'une confrontation, d'un face-à-face des cultures. Peu importe le lieu... (Mabanckou 2012 : 159)

Pour ce faire, une des voies à explorer est une Afrique multilingue qui s'écrira dans les langues autochtones et dans les langues occidentales, qui donnera lieu à une "création déterritorialisée" (Mabanckou et Le Bris 2013: 9). L'Afrique à venir est désormais multiple, complexe et en pleine mutation, produisant une sorte de polythéisme social et culturel ; elle apparait sous la forme d'un assemblage d'espaces produits sur le mode de l'imbrication et de la circulation. L'être africain implique la participation à cette déclosion $\mathrm{du}$ continent et du monde. À la question: "L'émigration, a-t-elle influencé votre écriture?", Alain Mabanckou confirme qu'il s'agit de dépasser les frontières en tant qu'écrivain et en tant qu'être humain :

Lorsqu'on me demande si l'émigration influe sur mon écriture, il m'est impossible de donner une réponse précise et définitive. Sans doute parce que je suis de plus en plus persuadé que le déplacement, le franchissement des frontières, nourrit mes angoisses, contribue à façonner un pays imaginaire qui, finalement, ressemble à ma terre d'origine. Il y va de ma propre quête intérieure, de ma façon de concevoir l'univers. J'ai choisi de ne pas m'enfermer, de prêter l'oreille au bruit et à la fureur du monde, de ne jamais considérer les choses de manière figée. (Mabanckou 2012: 131)

11 Alain Mabanckou a été le premier écrivain africain à occuper la chaire de création artistique du Collège de France. Cette entrée symbolique d'un écrivain africain dans l'élite de la langue de Molière et de Kourouma représente une ouverture au monde et situe l'écrivain francophone africain au cœur de sa modernité. Felwine Sarr remarque le rôle de ces écrivains et leurs contributions pour changer les systèmes et les structures mentales occidentales. Cette expérience nouvelle de l'ici et de l'ailleurs demeure un point clé :

Les écrivains africains vivant dans la diaspora ont posé un regard sur le continent à partir de l'ailleurs de leur exil. Leur lieu d'ancrage les a amenés à penser la synthèse culturelle, les identités nomades et circulaires, mais aussi à rêver et à fantasmer l'Afrique. [...] Abdourahman Waberi imagine une Afrique devenue puissante et prospère, et autour de laquelle s'agglutinent Eurasiens et Américains miséreux et désireux de s'y installer. Léonora Miano pense l'Afrique et le projet d'y édifier notre humanité avec ses ombres et ses clartés, mais elle interroge aussi les nouvelles constructions identitaires en terre européenne, sur fond de mémoire, d'oubli et de réminiscences. Cette Afrique-là, qui pose un regard de loin, a son mot à dire dans la construction de l'Afrique qui arrive. (Sarr 2016 : 135) 


\section{Léonora Miano : l'identité frontalière et l'afrodescendance}

12 Léonora Miano rassemble dans deux essais une panoplie de thèmes propres à sa sensibilité d'écrivaine. Il s'agit pour elle d'une invitation à mieux lire les écrivains subsahariens d'hier et surtout d'aujourd'hui. Habiter la frontière (2012) et L'Impératif transgressif (2016) offrent le substrat politique et esthétique de sa poétique. Ces textes fournissent une meilleure connaissance de l'auteure, qui se dit contribuer «à la réhabilitation de la conscience de soi au sud du Sahara (2012:17) et lui ont permis d'être remarquée parmi les africanistes qui élaborent une nouvelle pensée sur l'Afrique subsaharienne.

Un des thèmes abordés est le rapport à la langue non seulement en situant la langue française au centre, mais également en jouant sur les « autres langues » - autochtones ou pas, comme le cas de l'anglais - et sur le(s) sujet(s) parlant(s) à qui elle renvoie la capacité de se dire: «Aux subsahariens de continuer à s'emparer du français pour dire ce qui fut longtemps tu, la langue étant parfaitement neutre quant à sa capacité à dire le vrai » (Miano 2016a: 104). Pour elle, l'Afrique subsaharienne francophone est un espace multilingue « où la langue française est traversée par d'autres, imprégnée par d'autres imaginaires, rythmes et perceptions » (Miano 2016a : 94).

Son constat est qu'il faut repenser la notion de francophonie, telle qu'elle a été conçue depuis ses origines, soulignant "l'universalité de la langue française», tel que le proclamait, par exemple, Rivarol en 1784 dans son Discours sur l'universalité de la langue française :

Le temps est venu de dire le Monde Français, comme autrefois le Monde Romain, et la Philosophie, lasse de voir les hommes toujours divisés par des Maîtres qui ont tant d'intérêt à les isoler, se réjouit maintenant de les voir, d'un bout de la terre à l'autre, se former en République sous la domination d'une même langue ${ }^{13}$.

L'autre constat à faire est que : «Écrire en français n'est pas écrire français 》 (Miano 2012 :

29) et que : « La langue française telle que parlée sous le Sahara, n'est plus tout à fait celle que connaissent les Français » (Miano 2016a : 21). Par conséquent :

Les francophonies littéraires d'Afrique subsaharienne démontrent bien que la langue usitée, l'expression qui en découle, ne sont, en réalité, pas françaises. Non seulement écrire en français n'est-il pas écrire français, mais écrire en français n'implique même pas d'avoir, au préalable, une culture littéraire française. (Miano 2016a : 95)

$16 \mathrm{Si}$ « [u]ne partie de l'Afrique subsaharienne est dite francophone parce que le français y est la langue de pouvoir, aucunement en raison de sa pratique effective pour la majorité de la population" (Miano 2016a: 98), il faudrait changer le statut de la langue française qui passerait de «langue de domination » à « langue de partage ». Contre la francophonie institutionnelle qui considère les francophones d'Afrique subsaharienne comme de petits satellites devant graviter autour du soleil France, Miano proclame une troisième voie.

Contre la Francophonie institutionnelle hégémonique, une internationale francophone devrait permettre de se rapprocher les uns des autres, de bâtir sur le continent, de meilleurs systèmes d'échanges, afin de forger un panafricanisme et d'envisager le français comme un lien avec les diasporas francophones d'Europe et des Amériques au sens large 
(Miano 2016a : 98). Pour Miano, un des instruments pour décoloniser le français, serait la création d'une nouvelle voix, nommée l'afrophonie (2016a : 99).

L'afrophonie est la mise en dialogue, par les Subsahariens et les Afrodescendants ${ }^{14}$ de leurs expériences. Elle est le lieu où naissent de nouvelles relations, où se déroulent les échanges et collaborations dont ils furent privés, l'espace intellectuel et créatif, au-delà du stigmate. [...] La désaliénation ne consiste pas en la répudiation de tout élément venu d'Occident, mais dans le choix du rôle à lui attribuer, dès lors qu'il a été identifié comme utile. Il est tout à fait possible d'avancer dans le sens de soi-même, de passer par la porte ouverte du français pour partager d'autres langues entre Subsahariens, avec les Afrodescendants et l'humanité dans son ensemble ${ }^{15}$. (Miano 2016a : 100)

L'afrophonie est conçue ainsi, à partir d'une position anti-hégémonique, qui implique un usage transgressif de la francophonie lié au concept de frontière, à partir d'une vision subsaharienne, qui en fait un lieu de médiation, de rencontre, en aucun cas de rupture. Loin d'attribuer des reproches univoques à la France et aux Français de l'Hexagone, l' Afrique qui vient, dont l'une des langues d'écriture sera le ou les français, a une tâche qui n'est attribuable qu'aux Subsahariens, à qui notre auteure exige une responsabilité partagée.

Léonora Miano se définit elle-même à partir de la notion de frontière : «J'écris dans l'écho des cultures qui m'habitent: africaine, européenne, africaine américaine, caribéenne » (2012 : 144), nous dit-elle. Elle défend le concept d'identité frontalière, notion qui recoupe à la fois une préoccupation identitaire, un positionnement dans un monde en mouvement ou encore une volonté esthétique.

Son afropéanisme se construit sur la même dynamique de frontière. Non plus ligne de rupture mais « espace d'accolements » (Miano 2012 : 25), celle-ci est le lieu de naissance de sujets culturels complexes, nourris par leurs parcours individuels internationaux: Afrodescendants arrivés ou nés en Europe. Ils appartiennent aux deux continents sans que cela leur coûte l'abandon ou le rejet de leur africanité (Miano 2012 : 84). On pourrait dire de l'afropéanisme de Léonora Miano: «qu'il est une négociation constante de la frontière » (Lefilleul $2014: 85$ ).

Je suis une Afro-occidentale parfaitement assumée, refusant de choisir entre ma part africaine et ma part occidentale. [...] Il me semble que ce que j'affirme pour moi-même est bel et bien la réalité actuelle de ma terre natale. [...] être africain, de nos jours, c'est être un hybride culturel. C'est habiter la frontière. (Miano 2012 : 28)

Miano ainsi que Mbembe convoquent une variété de cultures et de notions conceptuelles et mettent tous deux l'accent sur la circulation des identités et des imaginaires culturels. Leurs discours s'articulent autour d'éléments clés semblables : la pensée de la circulation comme support de la construction identitaire, le caractère historique et surtout précolonial de la mobilité en Afrique, l'aspect cosmopolite de cette circulation humaine et culturelle, et enfin la nécessité de repenser l'africanité et de placer le geste créatif au cœur de cette préoccupation.

L'afropéanisme comme l'afropolitanisme sont la continuité d'une mobilité présente sur le continent africain qui se produit avant la présence coloniale. Achille Mbembe et Léonora Miano insistent tous deux sur le caractère historique de cette circulation. Ils nous rappellent que le processus de créolisation des cultures africaines a commencé bien avant la colonisation, que les échanges culturels et commerciaux y avaient largement cours et se sont ensuite déplacés avec l'arrivée des Occidentaux. La frontière et la fixité qui en découlent sont des faits coloniaux. Miano, en choisissant de redéfinir la frontière, 
déconstruit l'imposition d'une fracturation géographique et culturelle pour définir les identités multiples de ce qu'elle nomme les "Afropéens ${ }^{16}$ ", et plus largement les "Afrodescendants ${ }^{17}$ ». Les identités proposées par l'afropolitanisme et l'afropéanisme sont des identités multiples, cosmopolites, façonnées par la rencontre et l'hybridité.

Tous deux ancrent leurs réflexions dans le continent africain et sa diaspora contemporaine. Ils choisissent de mettre en lumière la réalité multiculturelle de ces deux entités. C'est la mobilité des individus qui provoque (et a provoqué) ce cosmopolitisme. Dans le cas de Léonora Miano, ses textes de fiction évoquent un espace transnational où il existe une absence de localisation dans un espace littéraire identifiable, et où la marque spatiale reste floue. Dans son dernier roman, Crépuscule du tourment (2016b), les personnages vivent leur parcours existentiel entre les deux continents - l'Afrique et l'Europe -, le Nord évoquant une éventuelle France ou Europe.

Écrire la frontière implique une vision particulière du genre. Elle, qui refuse l'étiquette de «féministe » ou "féminine », affirme : «si le féminisme consiste à faire valoir les droits d'une catégorie outrée, il va sans dire que je le défends » (Miano 2012 : 34). Elle insiste sur le fait que ses romans parlent d'humanité et, qu'une fois dépassée la question biologique, le masculin et le féminin ne sont que des constructions sociales et culturelles.

Mais la cause des femmes demeure très présente tout au long de ses textes. Elle y offre, par exemple, une vision particulière de la « soumission » des femmes subsahariennes aux canons de beauté esthétique occidentaux, «inaccessible horizon des femmes du monde entier ». La recherche d'une chevelure lisse qui glisse entre les doigts est une des préoccupations majeures des femmes subsahariennes qui fréquentent les salons de beauté afro afin de trouver le meilleur produit lissant et nourrissant. Cette recherche permanente et inassouvie est pour l'auteure, qui « milite pour cette fameuse marque qui permet aux femmes de souche subsaharienne de se réconcilier avec leur héritage », la métaphore de l'aliénation de la femme africaine.

Son dernier roman, Crépuscule du tourment, donne voix à quatre femmes, la Mère, l'épouse, l'amante et la sœur, destinées au même homme. L'univers féminin est explicitement nommé à travers les personnages qui, bien que différents du point de vue social et générationnel, se cherchent et se retrouvent autour de la «magie féminine " de l'écrivaine féministe afro-américaine Ntozake Shange (New Jersey, 1948), Where there is a woman there is magic:

J'ai lu cela il y a des années, je me suis dit bof, cela ne m'impressionnait guère, cela me contrariait même un peu, ces femmes qui en faisaient tout un plat de leur féminité, comme si c'était un truc spécial, je voyais ma mère, je me voyais moi et ne comprenais pas, à présent je sais qu'il y a du vrai, le texte disait aussi qu'il fallait connaître sa propre magie pour la rendre effective, j'irai débusquer la mienne. (Miano 2016 : 183)

Léonora Miano explore dans ses romans et dans ses textes théoriques la déportation transatlantique des Subsahariens, désignée aussi comme Traite des Noirs, Traite des esclaves (Miano 2016a: 141). Ce thème parcourt sa fiction et ses personnages en portent l'empreinte. Le drame fondateur - nommer la douleur- (Miano 2016a: 155) de la déportation et de la traite est à la base ; il s'agira donc d'une réhabilitation du passé « au besoin de mieux saisir le présent à la recherche d'une voie permettant de se dessiner un avenir » (Miano 2016a : 67).

L'entreprise de Miano vise la restitution et la réhabilitation du point de vue subsaharien de cette période historique non seulement dans des travaux académiques ou dans les 
discours médiatiques mais aussi dans l'imaginaire français et afrodescendant ${ }^{18}$. D'autre part, la révision, la réhabilitation et la réparation sont les notions autour desquelles l'auteure déploie son militantisme dans la création littéraire. Ce sont les sociétés subsahariennes les premières qui doivent se raconter le passé. Elle propose de mettre en place des synergies entre chercheurs et créateurs, pour aider les populations subsahariennes à se réapproprier leur histoire, à reprendre la parole :

Parler, représenter, expliquer, nommer, c'est aussi consoler. L'Afrique subsaharienne pour qui le rire est une marque d'élégance et de pudeur, doit s'autoriser à pleurer. Il ne s'agit pas que ces larmes, encore refoulées, prennent la forme de récriminations adressées aux puissances européennes, ou d'un ressentiment corrosif. (Miano 2016a: 169)

Quelques pages plus loin elle écrit: "C'est d'une conscience de soi assainie qu'il est question : ni complaisance, ni autoflagellation » (174).

Alain Mabanckou et Léonora Miano pratiquent dans leurs essais une réflexion sur l'identité du sujet africain, anciennement colonisé et racialisé, et de façon indirecte, une réflexion sur la littérature africaine de langue française comme une éventuelle réponse à la démarche identitaire. Cette réflexion ne se fait ni à partir du continent africain, ni pour le continent, mais au sein d'une mondialisation où l'Afrique et ses diasporas se rencontrent, s'interpellent et s'enrichissent. Tous les deux ne sont pas dupes et ils sont conscients de la complexité de ce processus de réflexion et de transformation qui rejoint les postulats de Mbembe et de Sarr et qui implique désormais un travail de réécriture et de dé-victimisation de soi. Nous nous trouvons face à un rapport entre la France et l'Afrique qui se fait par le biais de la création artistique où la médiatisation de la littérature s'offre comme un nouvel espace de réflexion pour les spécialistes ou les africanophiles, mais également pour un large public qui s'intéresse à la matière humaine.

\section{BIBLIOGRAPHIE}

Balibar, Étienne et Wallerstein, Immanuel, 1997, Race, nation, classe : les identités ambiguës, Paris, La Découverte.

Casanova, Pascale, 1999, La République mondiale des lettres, Paris, Seuil.

Imorou, Abdoulaye, 2017, « Le nouveau discours africain, version bêta », Études littéraires africaines , no 43, p. 145-151, [En ligne], https://id.erudit.org/iderudit/1040923ar, consulté le 16 juillet 2019. Doi : 10.7202/1040923ar

Lefilleul, Alice, 2014, « Afropéanisme, identités frontalières et afropolitanisme. Penser les nouvelles circulations », Africultures, $\mathrm{n}^{\circ}$ 99-100 (2014/3), p. 84-91.

Mabanckou, Alain, 2007, Lettres à Jimmy, Paris, Fayard.

Mabanckou, Alain, 2012, Le Sanglot de l'homme noir, Paris, Fayard.

Mabanckou, Alain et Le Bris, Michel, 2013, L'Afrique qui vient. Anthologie, Paris, Hoëbeke. 
Mabanckou, Alain, 2016a, Lettres noires : des ténèbres à la lumière, Leçons inaugurales du Collège de France, Paris, Collège de France, [En ligne], http://books.openedition.org/cdf/4413, consulté le 16 juillet 2019 .

DOI : $10.4000 /$ books.cdf.4413

Mabanckou, Alain, 2017a, « À propos du Sanglot de l'homme noir », dans A. Mabanckou (dir.), Penser et écrire l'Afrique aujourd'hui, Paris, Seuil, p. 121-134.

Mabanckou, Alain (dir.), 2017b, Penser et écrire l'Afrique aujourd'hui, Paris, Seuil.

Mangeon, Anthony, 2017, « Répétition générale », Études littéraires africaines, n 43, p. 141-145, [En ligne], https://id.erudit.org/iderudit/1040922ar, consulté le 16 juillet 2019

DOI : 10.7202/1040922ar

Mbembe, Achille, 2005, « Afropolitanisme », Africultures, [En ligne], http:// www.africultures.com/php/?nav=article\&no=4248, consulté le 16 juillet 2019.

Mbembe, Achille, 2006, «Qu'est-ce que la pensée postcoloniale ?», Esprit, décembre 2006, p. 117-133.

DOI : $10.3917 /$ espri.0612.0117

Mbembe, Achille, 2010a, Sortir de la grande nuit. Essai sur l'Afrique décolonisée, Paris, La Découverte. Mbembe, Achille, 2010b, « Pièce d'identité et désirs d'apartheid » dans M. Le Bris et J. Rouaud (dir.), Je est un autre, Paris, Gallimard, p. 115-122.

Mbembe, Achille, 2010c, "La République et l'impensé de la "race" ", dans N. Bancel, F. Bernault, P. Blanchard, A. Boubeker, A. Mbembe et F. Vergès (dir.), Ruptures postcoloniales, Paris, La Découverte, p. 205-216.

Mbembe, Achille, 2017, « L'Afrique qui vient », dans A. Mabanckou (dir.), Penser et écrire l'Afrique aujourd'hui, Paris, Seuil, p. 17-31.

Miano, Léonora, 2012, Habiter la frontière, Paris, L'Arche.

Miano, Léonora, 2016a, L’Impératif transgressif, Paris, L'Arche.

Miano, Léonora, 2016b, Crépuscule du tourment, Paris, Grasset.

Miano, Léonora, 2017, « De quoi Afrique est-il le nom ? », dans A. Mbembe et F. Sarr (dir.), Écrire l'Afrique-Monde, Dakar, Philippe Rey, Jimsaan, p. 101-115.

Sarr, Felwine, 2016, Afrotopia, Paris, Philippe Rey.

Waberi, Abdourahman, 2017, « Les enfants de la postcolonie, précédé d'une note liminaire », dans A. Mabanckou (dir.), Penser et écrire l'Afrique aujourd'hui, Paris, Seuil, p. 148-161.

\section{NOTES}

1. La pensée circulaire de Mbembe s'insère dans sa définition de la pensée postcoloniale qui se caractérise par son hétérogénéité. Cette pensée éclatée, en mouvement, est loin de la pensée binaire et linéaire. Il s'agit d'une démarche intellectuelle face aux défis d'un monde globalisé. La pensée circulaire est la pensée de l'enchevêtrement et de la concaténation. L'identité s'origine dans la multiplicité et la dispersion. Par exemple, la colonisation n'apparaît plus comme une domination mécanique et unilatérale qui force l'assujetti au silence et à l'inaction. Au contraire, le colonisé est un individu vivant, 
parlant, conscient, agissant, dont l'identité est le résultat d'un triple mouvement d'effraction, de gommage et de réécriture de soi (Mbembe 2006).

2. Il pourrait paraitre paradoxal de défendre une ouverture en africanisant ou particularisant des notions générales - afropolitisme pour cosmopolitisme. Il s'agit de rester fidèle à la théorie postcoloniale et de faire de la « question africaine » une question planétaire. En africanisant certains concepts, le propos est un changement de « logiciel » (Imorou $2017: 150$ ) qui vise à faire comprendre qu'il n'y a plus de question africaine ou diasporique qui ne renvoie en même temps à une question planétaire. Parler d' afropolitisme au lieu de cosmopolitisme ne serait que la réponse osée afin d'inverser la logique occidentale où universalisme et Occident vont de pair.

3. À noter que Mbembe, Mabanckou ou Sarr dépassent l'espace littéraire et intellectuel stricto sensu. Ils ne négligent point la projection médiatique et le potentiel divulgateur de leurs idées. Les Ateliers de la pensée sont diffusés sur Internet. Mabanckou se montre très actif sur Facebook et Twitter et il n'hésite pas à critiquer la politique francophone du président Macron ou l'actualité politique du Congo. Par exemple, si Mabanckou refuse le projet de francophonie, Sarr accepte la mission sur la restitution des biens culturels à l'Afrique.

4. Cette graphie rassemble en un seul terme les deux acceptions du mot: Francophonie, avec un « F » majuscule représente les Institutions de l'OIF (70 États et gouvernements) et renvoie au statut institutionnel de la France et à son projet de diffusion de la langue et de la culture française. Cette représentation institutionnelle est liée à la stratégie diplomatique de la France et à sa présence en Afrique suite à l'héritage colonial. La francophonie, avec un « $\mathrm{f}$ » minuscule, rassemble les millions de francophones dispersés dans le monde. Au-delà des notions géographiques politiques, d'un point de vue linguistique, la francophonie renvoie à la pratique effective de la population, aux multiples appartenances que l'on peut avoir avec la langue française, dans un contexte monolingue ou multilingue, ainsi qu'au partage d'un «espace francophone" lié à une culture, tel que l'on parle de "culture latine ", par exemple. Il est à remarquer que les francophones ne partagent ni un imaginaire culturel homogène, ni l'imaginaire culturel exclusivement français.

5. Léonora Miano souligne que la révision et la réappropriation de la Déportation transatlantique des Subsahariens (DTS) doit sortir de l'étau académique et des cercles des spécialistes. Par exemple la littérature africaine d'expression française, dont elle fait preuve, doit viser un public, les Afrodescendants européens.

6. Par nouveau discours africain, je parle du discours sur l'Afrique indépendamment des origines des auteurs ou de leurs lieux d'écriture.

7. Léonora Miano a reçu le Prix Fémina en 2013 pour son roman, La Saison de l'ombre; Alain Mabanckou a remporté le Prix Renaudot en 2006 avec son roman Mémoires de porcépic, et a été sélectionné pour le Prix Goncourt en 2015 avec son roman Petit Piment.

8. Cette anthologie rassemble des auteurs variés, de langues et de lieux différents et dont le dénominateur commun est un lien d'appartenance avant tout intime au continent africain. On y lit - entre autres - Léonora Miano, Wilfrid N’Sondé, Mia Couto, ou Mackenzy Orcel, écrivain haïtien. La couleur de peau, le lieu de vie, les sujets de prédilection sont variés et ne prédisposent donc pas l'écrivain à un étiquetage « africain ». 
9. Voir les articles de Mangeon et Imorou, à propos de ce volume collectif dans Études littéraires africaines, $\mathrm{n}^{\circ}$ 43, 2017.

10. «Depuis 2000, sa croissance économique est supérieure à $5 \%$. Parmi les taux de croissance les plus élevés du monde de 2008 à 2013, les pays africains sont bien représentés (Sierra Leone 9,4\%, Rwanda 8,4 \% Éthiopie 8,4\%, Ghana 8,11\%, Mozambique $7,25 \%$ ). [...] D'autre part, la jeunesse africaine investit les réseaux sociaux, tient des blogs, tweete, commente l'actualité, exprime ses opinions et pose son regard sur la marche du monde. Elle est prompte à réagir contre la francophonie quand celle-ci se pare des vêtements de l'impérialisme culturel, en dépit des dynamiques démographiques et sociolinguistiques qui font $\mathrm{du}$ français une langue non seulement africaine, mais qui ne survivra et ne maintiendra son rang de langue internationale que grâce à la démographie du continent africain » (Sarr $2016: 49$ et 94).

11. Alain Mabanckou a écrit un premier essai, Lettre à Jimmy, consacré à l'écrivain James Baldwin dans une sorte d'hommage épistolaire contemporain à l'auteur et son combat. Tous deux refusent les identités étatiques ne reconnaissant que deux identités : celle d'écrivain et celle d'être humain. En 2012, Mabanckou nous offre un deuxième essai, Le Sanglot de l'homme noir, faisant allusion au sanglot de l'homme blanc de Pascal Bruckner. Le travail d'intertextualité interpelle l'identité noire à partir de différents romans d'auteurs français et africains. Sa réflexion, à mi-chemin entre la dénonciation et l'autocritique est une invitation contre la complaisance et la victimisation.

12. Le défi ne s'avère pas facile. Il s'agit d'aller au-delà de l'essentialisme noir ainsi que de prendre acte de ce que l'Europe n'est qu'une province du monde, que l'Afrique n'est pas l'exemple de l'ailleurs ou que les régions du monde sont en relation.

13. Rivarol, De l'universalité de la langue française, 1784, [En ligne], https://gallica.bnf.fr/ ark:/12148/bpt6k98382.image, cité par Léonora Miano (2016a : 75).

14. Les Afrodescendants sont des Européens d'ascendance subsaharienne (Miano 2012 : 140). «Les Afrodescendants ont perdu leur arbre généalogique, pas leur identité, sauf à considérer qu'elle n'aurait à voir qu'avec le nom des aïeux. Leur culture est celle qu'ils ont créée, tout comme la culture française, aujourd'hui, est celle que façonnent les Français aujourd'hui. Les Afrodescendants n'ont jamais perdu l'Afrique, ils l'ont adaptée à leur espace, en ont conservé ce qui pouvait y faire sens » (Miano 2016a : 50).

15. La situation linguistique de beaucoup de pays francophones d'Afrique subsaharienne est multilingue, où la communication se fait à partir d'un brassage de toutes les langues. À noter, le cas du Cameroun : «les Camerounais ne se comprennent entre compatriotes que parce qu'ils ont inventé des langues véhiculaires comme le pidgin ou le camfranglais, et parce qu'ils se sont appropriés les anciennes langues coloniales » (Miano 2016a : 89).

16. «Par Afropéen, je n'entends pas parler d'une personne qui, comme moi, serait venue en Europe à l'âge adulte. Il s'agit, à l'inverse, de gens dont le vécu est essentiellement européen, que l'on peut définir comme Européens, et qui ont des attaches subsahariennes » (Miano 2016a : 55).

17. «Le mot Afrodescendant reconnaît et célèbre l'Afrique comme fondement identitaire essentiel, sinon unique. Il n'est pas la négation d'autres apports, mais la reconnaissance $\mathrm{du}$ fait que, sur les plans symboliques et intimes, les cultures en question présentent de nombreux traits subsahariens » (Miano $2012: 120$ ).

18. Voir à ce propos « De quoi Afrique est-il le nom? » (Miano 2017). 


\section{RÉSUMÉS}

Cet article analyse les écrits théoriques de deux écrivains qui participeraient à la mise en œuvre d'une « renaissance littéraire » africaine, à partir du militantisme manifeste dans leurs créations artistiques et des liens qu'ils tissent avec la critique africaniste contemporaine. Alain Mabanckou et Léonora Miano appartiennent à la « génération transcontinentale » de Waberi, et ils partagent des traits communs susceptibles de marquer un tournant par rapport aux générations précédentes et par rapport au rôle de la littérature tout en donnant une dimension politique flagrante à l'acte d'écriture. Il s'agit ici de rapporter les lignes d'écriture d'une Afrique qui vient, métaphore de l'éclosion à venir du continent, à la source de laquelle se trouve la création artistique. Si Mabanckou nous offre des réflexions littéraires proches de la pensée postcoloniale de Mbembe, Miano projette un rapport nouveau à l'Afrique à partir de l'identité frontalière et de l'afrodescendance. Nous sommes face à de nouvelles expressivités littéraires, à travers d'autres supports textuels (magazines spécialisés, réseaux sociaux, etc.) et visant d'autres destinataires, qui placent l'acte d'écriture et la figure de l'écrivain au centre d'un nouveau discours critique. Une nouvelle conception de la f(F)rancophonie ou un rapport autre face à la langue d'écriture se retrouvent à la source de la création littéraire. Ces deux écrivains proposent dans leurs textes une théorie de leur pratique fictionnelle. Tous les deux abordent des thèmes qui sont au cœur des rapports entre la France et l'Afrique, entre autres, la révision du statut d'immigré - et des Afrodescendants - et la réappropriation de l'histoire et de l'imaginaire des peuples colonisés par l'Europe occidentale.

This article analyses the theoretical writing of two writers who participated in the African francophone literary renaissance. Their works are influenced by their activism and their strong ties to African contemporary scholarly critique. Alain Mabanckou and Léonora Miano belong to what Waberi has termed the "Transcontinental generation." Their shared common features seem to represent a break away from previous generations and the role they gave literature. This new generation's writing process is given a blatant political dimension: they seek to report in writing "an Africa to come" (une Afrique qui vient), a metaphor that symbolises the imminent flourishing of the continent, enabled by artistic creation. Mabanckou's literary reflections echoe Mbembe's postcolonial approach while Miano foregrounds a new relationship with the continent, that of border-crossing identities and Afrodescendents. New forms of literary expressions have appeared (specialised magazines, social networks, etc.) that address other publics. They place the writing process and the figure of the writer at the centre of critical discourse. The concept of $f$ (F)rancophonie and the relationship to the language one writes in are being rethought and represent building blocks for literary creation. Both writers's texts offer theorisation of their fiction writing. They explore topics that are at the core of the relationship between France and Africa, such as the revision of immigrants' status-the Afrodescendents-and the reappropriation of the history and imagination of formerly colonized people. 
INDEX

Mots-clés : littérature africaine, identité, afropolitanisme, frontière, critique africaniste, Mabanckou (Alain), Miano (Léonora)

Keywords : African literature, identity, afropolitanism, frontier, African critique, Mabanckou (Alain), Miano (Léonora)

\section{AUTEUR}

JOSEFINA BUENO ALONSO

Université d'Alicante 